\title{
Silicon-nitride waveguides for on-chip Raman spectroscopy
}

\author{
Ashim Dhakal*a,b $^{\text {a, }}$ Pieter Wuytens ${ }^{\mathrm{a}, \mathrm{b}, \mathrm{c},}$, Frederic Peyskens ${ }^{\mathrm{a}, \mathrm{b}}$, Ananth Z Subramanian ${ }^{\mathrm{a}, \mathrm{b}}$, Nicolas Le \\ Thomas $^{\mathrm{a}, \mathrm{b}}$, Roel Baets ${ }^{\mathrm{a}, \mathrm{b}}$ \\ ${ }^{a}$ Photonics Research Group, INTEC-department, Ghent University - imec \\ ${ }^{\mathrm{b}}$ Center for Nano- and Biophotonics, Ghent University \\ ${ }^{c}$ Department of Molecular Biotechnology, Ghent University, Ghent, Belgium \\ Sint-Pietersnieuwstraat 41, B-9000 Ghent, Belgium \\ *Email: ashim.dhakal@intec.ugent.be
}

\begin{abstract}
The evanescent tail of the guided modes can efficiently excite Raman active molecules located in the cladding of a waveguide. Similarly, a significant fraction of the total emitted Stokes power is evanescently coupled to the same mode. Further, the enhancement effects inherent to the waveguide, alongside with the long interaction length, lead to an increased light-matter interaction, resulting in a higher sensitivity as required by spectroscopic applications, especially in the context of Raman spectroscopy. We calculate the spontaneous Raman scattering efficiency as a function of siliconnitride strip waveguide dimensions and show that under typical conditions, the overall efficiency is approximately two orders of magnitude higher than in confocal configuration in the free space. We also report the experimental demonstration of the use of silicon-nitride based photonic waveguides in a lab-on-a-chip context for Raman spectroscopy. To the best of our knowledge, this is the first demonstration of Raman spectroscopy using photonic waveguides.
\end{abstract}

Keywords: Raman spectroscopy, silicon-nitride waveguides, integrated photonics, lab-on-a-chip

\section{INTRODUCTION}

Raman spectroscopy is a key technology for the detection of a wide variety of atoms and molecules in applications ranging from physics and chemistry to biology and environmental sciences. As compared to many other types of sensors Raman spectroscopic techniques have the advantage that their selectivity is based on the specific spectrum rather than from chemical affinity of a given molecule to a functionalized surface. In spite of these advantages Raman spectroscopic techniques often require bulky or expensive instrumentation. If key parts of the optical functionality could be integrated on a chip, the route would be opened towards miniaturized low cost systems, with the potential of massive parallelism and multiplexing $[1,2]$.

Silicon photonics technology makes use of the technologies available in an advanced CMOS fab to fabricate photonic integrated circuits. Since silicon is only transparent above $1.1 \mu \mathrm{m}$, the technology is not readily suitable for applications requiring shorter wavelengths, as is often the case in biology. But by making use of silicon-nitride $\left(\mathrm{Si}_{3} \mathrm{~N}_{4}\right)$ waveguide layers instead of silicon, the technology can be extended to the entire visible and near infrared wavelength range. This is particularly relevant for Raman spectroscopy and fluorescence spectroscopy.

The value of using optical chips for Raman sensing is beyond the miniaturization, cost and reliability advantage associated with the use of mature wafer-scale technologies. There are also performance advantages. One important element is the fact that the high refractive index contrast in a $\mathrm{Si}_{3} \mathrm{~N}_{4}$ waveguide helps to enhance the electric field strength of a guided mode for a given mode power [3]. This is beneficial both for the absorption rate of the guided mode by a particle seen by the enhanced field and for the enhancement and coupling of light emitted by a particle coupled to the guided mode. Compared to free-space spectroscopy, the enhancement effects inherent to the waveguide, alongside with the long interaction length, lead to an increased light-matter interaction, resulting in a higher sensitivity as required by 
spectroscopic applications, especially in the context of Raman spectroscopy [4]. This enhancement can be boosted further through either resonant cavity enhancement and/or plasmonic enhancement by means of metallic nanostructures in the vicinity of the waveguides [5]. Furthermore light coupled to a single waveguide mode has a perfect spatial coherence and can in principle be decomposed in its spectral components with unlimited resolution.

In this paper, we summarize the technological, theoretical and experimental results to realize Raman sensors based on $\mathrm{Si}_{3} \mathrm{~N}_{4}$ photonic waveguides. In section 2 we shortly describe the key optical properties, such as waveguide-loss and the background luminescence of the $\mathrm{Si}_{3} \mathrm{~N}_{4}$ material used in our research. Section 3 summarizes the theoretical results on the expected overall efficiency of excitation and collection for molecules dispersed uniformly in the water cladding of the single mode waveguide with a single mode $\mathrm{Si}_{3} \mathrm{~N}_{4}$ waveguide. In section 4 we report the key experimental results.

\section{SILICON-NITRIDE WAVEGUIDE PLATFORM AND ITS SCATTERING UNDER RAMAN MICROSCOPE}

The wavelength range of 750-1200 $\mathrm{nm}$ is of specific interest in bio-sensing applications since it is delimited by the protein absorption at shorter visible wavelengths $(<750 \mathrm{~nm})$ and water absorption towards longer wavelength $(>1200$ $\mathrm{nm})$ [24]. Due to this reason, in this band the photo-damage by lasers to living cells is minimal. Although silicon-oninsulator (SOI) technology is a well-established technology making use of the tools available in an advanced CMOS fab to fabricate photonic integrated circuit, silicon is only transparent above $1.1 \mu \mathrm{m}$. Hence, the technology is not readily suitable for applications operating in the 750-1200 wavelength range requiring shorter wavelengths. But by making use of $\mathrm{Si}_{3} \mathrm{~N}_{4}$ waveguide layers instead of silicon, the technology can be extended to the entire visible and near infrared wavelength range. This is particularly relevant for Raman spectroscopy and fluorescence spectroscopy.

$\mathrm{Si}_{3} \mathrm{~N}_{4}$ is a versatile high index contrast platform that fulfills the above requirements for its transparency at both visible and near infrared wavelengths [6]. Besides it has low thermo-optic coefficient making it less sensitive to ambient fluctuations than for example silicon. These advantages make $\mathrm{Si}_{3} \mathrm{~N}_{4}$ a practical candidate for low-cost photonic devices and ultimately help in integrating the key functionalities required for lab-on-a-chip platform operating in the visible-NIR spectral window, especially Raman spectroscopic system on a chip level. Towards these ends, we have developed unclad low-loss $\mathrm{Si}_{3} \mathrm{~N}_{4}$ singlemode waveguides with loss below $1.5 \mathrm{~dB} / \mathrm{cm}[6]$ in the wavelength range 750-1000 $\mathrm{nm}$.

Furthermore, as studied with a confocal Raman microscope operating at $532 \mathrm{~nm}$ the Raman scattering and autofluorescence of the $\mathrm{Si}_{3} \mathrm{~N}_{4}$ material itself is low enough, thus enabling spectroscopy with their use. Figure 2 shows the spectrum of the waveguide stack (Figure 1, with $\mathrm{h}=220 \mathrm{~nm}, \mathrm{w}=\mathrm{slab}$ ) as acquired with a confocal Raman microscope, with pump wavelength at $532 \mathrm{~nm}$. We also see strong peaks at $520 \mathrm{~cm}^{-1}$ and $960 \mathrm{~cm}^{-1}$ due to silicon. We can also see a broad Raman emission in the range $2100-2400 \mathrm{~cm}^{-1}$ with a peak around $2330 \mathrm{~cm}^{-1}$. Z-scan with the confocal microscope and further experiments with the waveguides reveals that they are from silicon-nitride material as will be discussed in section 4 .

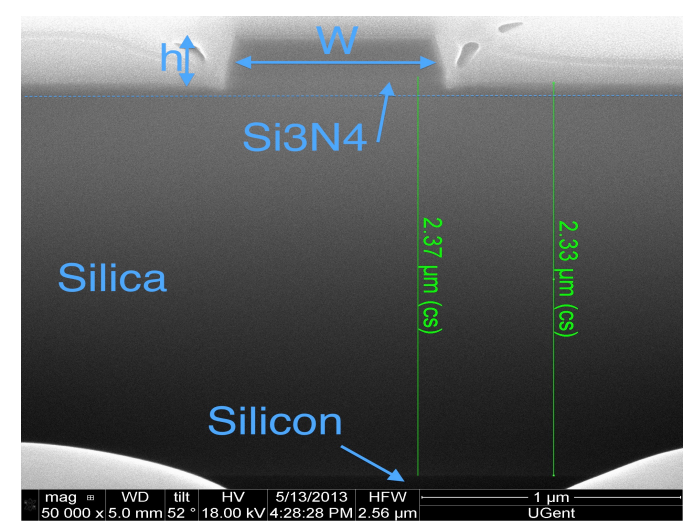

Figure 1: Cross section view of $\mathrm{Si}_{3} \mathrm{~N}_{4}$ waveguide discussed in this article. Typically we use $\mathrm{h}=220 \mathrm{~nm}$ and w= $700 \mathrm{~nm}$. 
Confocal Raman scan of a Si - $\mathrm{SiO}_{2}(2.4 \mu \mathrm{m})$ - $\mathrm{SiN}(220 \mathrm{~nm})$ stack

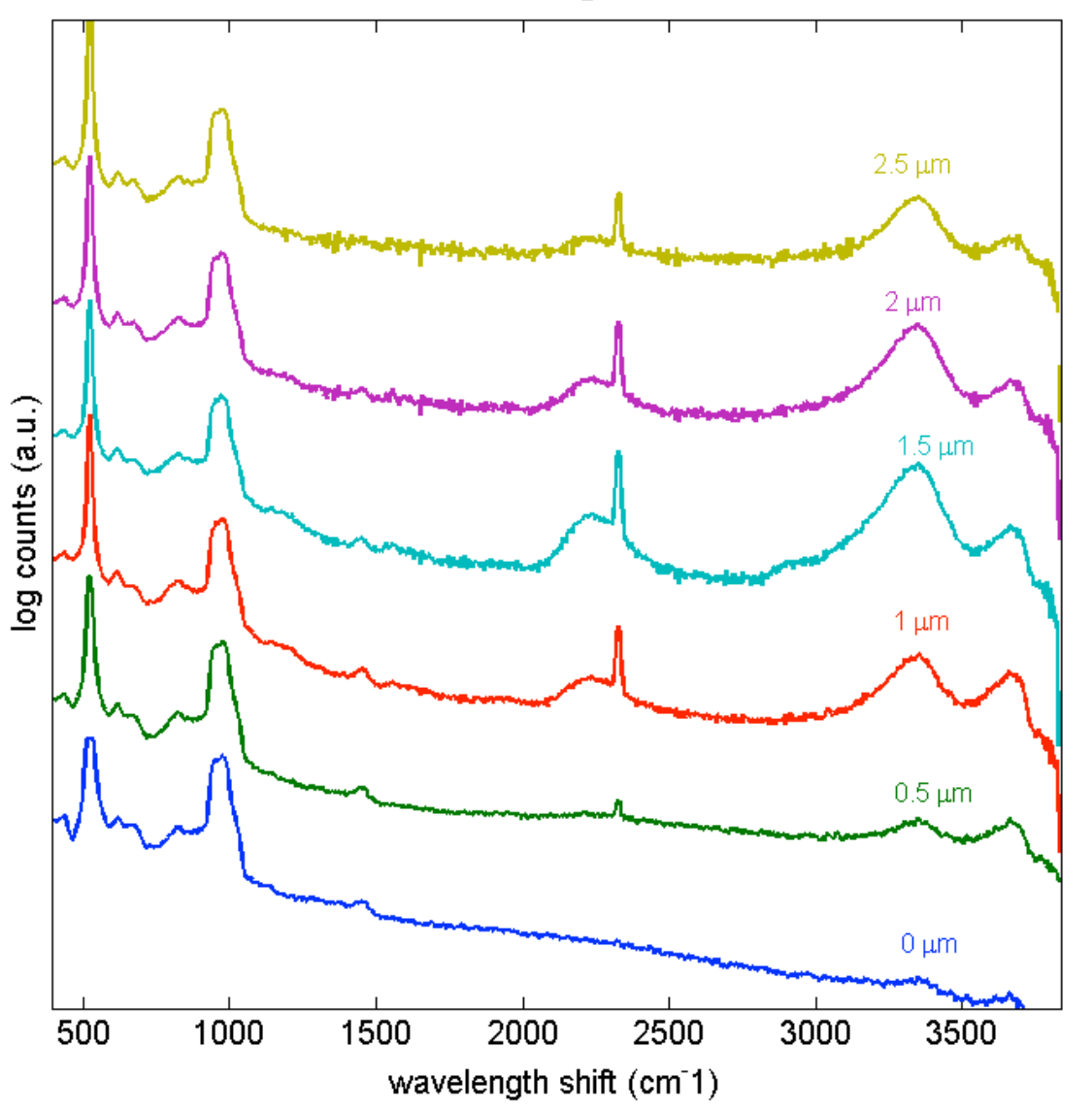

Figure 2: A confocal Raman z-scan of a $220 \mathrm{~nm}$ silicon-nitride top layer on $2.4 \mu \mathrm{m}$ silicon dioxide, deposited on a silicon-substrate shows the Raman contributions of the $\mathrm{Si}_{3} \mathrm{~N}_{4}$. On the silicon substrate (lowest curve), we see the well-known silicon Raman peaks at $520 \mathrm{~cm}^{-1}$ (satured) and $960 \mathrm{~cm}^{-1}$. When moving up in the material stack, we can identify specific Raman contributions of the siliconnitride: a broad Raman emission in the range $2100-2400 \mathrm{~cm}^{-1}$ with a peak around $2330 \mathrm{~cm}^{-1}$. These peaks were not present in a silicon dioxide-silicon material stack, confirming they originate from the silicon-nitride (data not shown). The spectra presented are offset for clarity, and were measured using a commercial confocal Raman microscope (WITec) with a 532 $\mathrm{nm}$ laser, focused through a $0.9 \mathrm{NA}$ air objective with $20 \mathrm{~mW}$ power on sample and a 60 second integration time.

\section{COLLECTION EFFICIENCY OF SPONTANEOUS RAMAN SIGNAL}

Recently, we investigated the scattering efficiency of a particle located in the vicinity of a channel waveguide for several geometries [4]. We theoretically determined the overall efficiency ( $\eta$ ) of (Raman) scattering from a guided mode and subsequent emission coupled back to the fundamental mode (both TE and TM) of a rectangular channel waveguide by uniformly distributed particles in its surrounding. We define $\eta$ as the ratio between the emitted power collected by the waveguide and the incident pump power in the mode. It was shown that $\eta$ is highly dependent on the mode area that further depends on the waveguide index and geometry. The same methodology can be applied to more popular strip waveguide geometry (Figure 1) for $\mathrm{Si}_{3} \mathrm{~N}_{4}$ waveguides [5]. In this section, we numerically evaluate $\eta$ as a function of the waveguide width $(w)$ for several thickness $(h)$ values for $\mathrm{Si}_{3} \mathrm{~N}_{4}(n=2)$ waveguides in water $(n=1.33)$ cladding. 
Assuming that the Raman emission can be modelled as the radiation of an oscillating dipole, and neglecting the Stokes shift, in the weak coupling regime, it can be shown that the efficiency of spontaneous Raman collection from molecules of scattering cross section $\sigma$ and density $\rho$ lying in the surrounding of a waveguide into a mode of a channel waveguide of length $l$ is given by [4]:

$$
\eta=\frac{\pi^{2} \lambda_{0}^{2}}{\left(\varepsilon_{0} \varepsilon\right)^{2} n}\left(\kappa n_{g}^{2} \Gamma^{2} l\right) \sigma \rho
$$

Where, $n_{g}$ is the group index of the mode, $n=\sqrt{\varepsilon}$ is the refractive index of the surrounding medium, and $\varepsilon_{0}$ is the permittivity of the vacuum, $\kappa$ is a constant relating the polarizability and the cross-section of the molecule, $\Gamma$ is a parameter dependent on the overlap of the mode area and the sensing area, given by:

$$
\Gamma^{2}=\int_{\text {sens }} d s\left(\frac{\varepsilon_{0} \varepsilon\left|E\left(\vec{r}_{0}\right)\right|^{2}}{\iint \varepsilon_{0} \varepsilon(\vec{r})|E(\vec{r})|^{2} d \vec{r}}\right)^{2}
$$

We use the COMSOL finite element eigenmode solver to determine the field of the waveguide modes for several waveguide geometries and solve Eq. (1). For simplicity, we neglect material dispersion, pump depletion, and assume $n_{\mathrm{g}}$ $\approx n_{\text {eff }}$ as this will have negligible impact. As an example, we take $\sigma=3 \times 10^{-30} \mathrm{~m}^{2} \mathrm{Sr}^{-1}$ molecule ${ }^{-1}$, as a typical cross-section of the dyes, $\rho=1 \mathrm{~mole} /$ liter and calculate $\eta$ for a $1 \mathrm{~cm}$ long waveguide. It is emphasized that the parameter values we have chosen do not affect the generality of our results because $\eta$ scales in a simple way with these parameters as per Eq. (1).

Figure 3 shows the calculated $\eta$ as a function of the waveguide width $(w)$ and height $(h)$ for $\mathrm{Si}_{3} \mathrm{~N}_{4}$ waveguides for bulk sensing for fundamental TE and TM polarized mode. The maximal efficiency, $\eta_{\max }$ is approximately $1.5 \times 10^{-8}$, implying that under ideal conditions, for $1 \mathrm{~mW}$ of pump power in the waveguide, $15 \mathrm{pW}$ of scattered signal can be collected by the guided mode. It is to be noted that the equivalent efficiency in a free-space confocal microscope system using diffraction-limited beams would be in the order of $10^{-10}$. The efficiency is maximal near the cutoff widths and decreases sharply before becoming almost constant for wider widths.

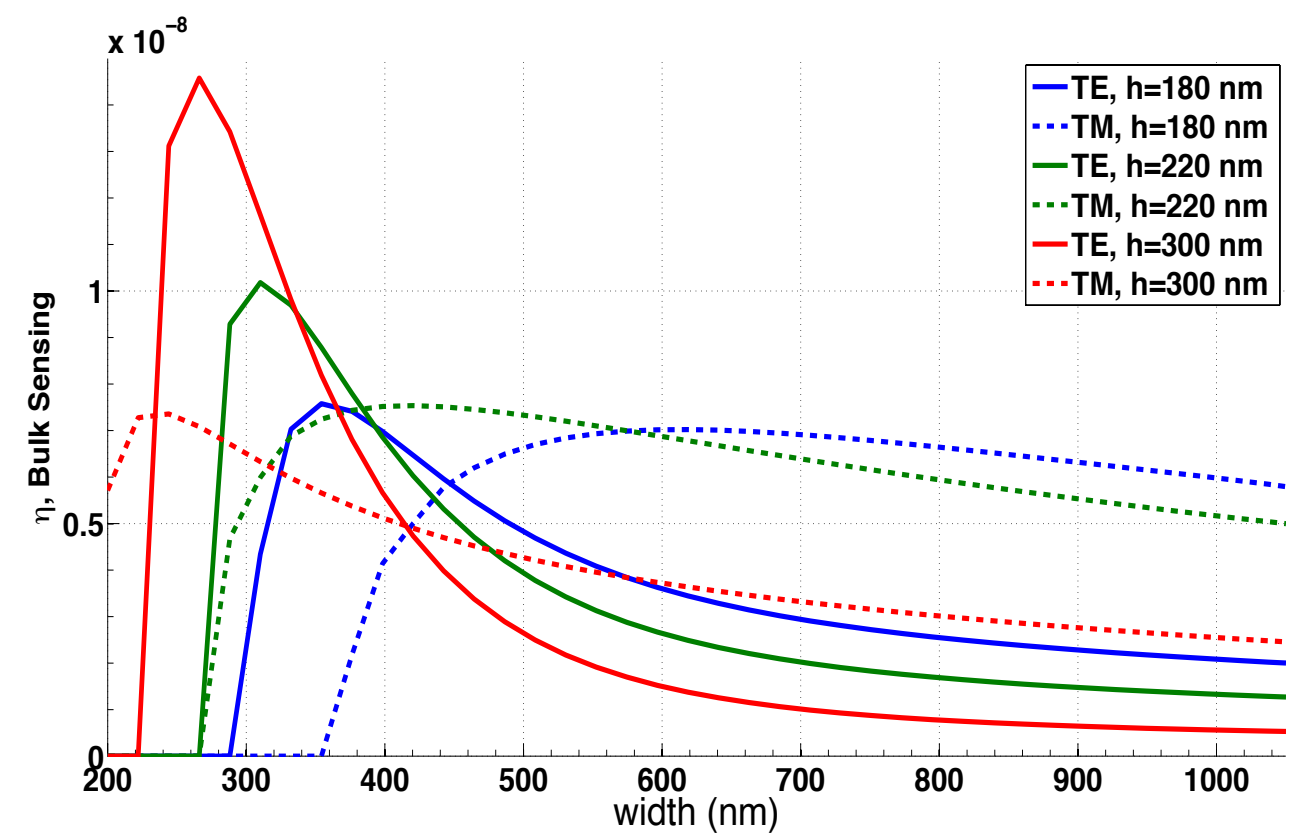

Figure 3: Efficiency of Raman signal collection for the fundamental TE and TM mode of $\mathrm{Si}_{3} \mathrm{~N}_{4}$ strip waveguides [5] as a function of waveguide widths for several heights (h) of the strip. 


\section{EXPERIMENTAL}

The experimental setup is illustrated in figure 4. A tuneable Ti-Sapphire CW laser $(\lambda=725-965 \mathrm{~nm})$ was used as the pump source. For the experiments, a wavelength of $785 \mathrm{~nm}$ is coupled to the waveguide by end-fire coupling using an aspheric lens of effective focal length $8 \mathrm{~mm}(\mathrm{NA}=0.5)$. The co-propagating Raman signal (Stokes) generated by the pump and collected by the waveguide is then collimated via another lens of focal length $6.5 \mathrm{~mm}(\mathrm{NA}=0.5)$ towards an edge filter, with the edge wavelength at $790 \mathrm{~nm}$ to block any stray pump light leaking into the spectrometer. The Raman signal is then focused to a single-mode optical-fibre using a parabolic mirror of $15 \mathrm{~mm}$ effective focal length (NA=0.2) and measured using a commercial spectrometer (Avantes SensLine).

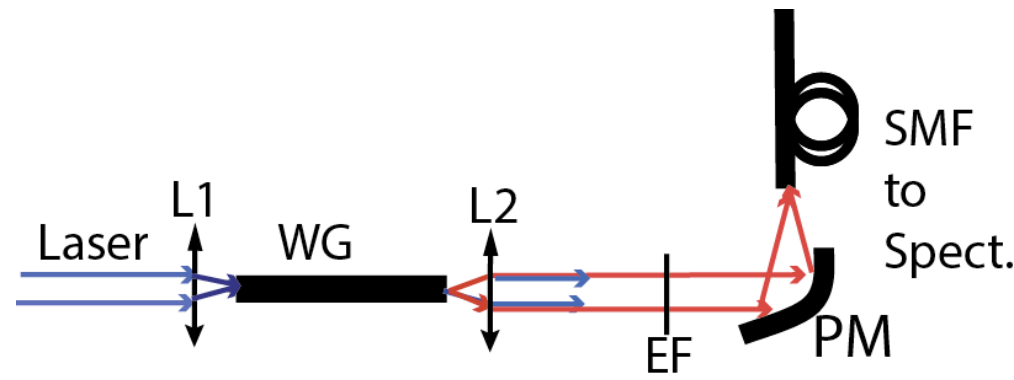

Figure 4: The schematic of experimental setup. The pump laser at $785 \mathrm{~nm}$ is focused at the waveguide (WG) facet using an aspheric lens (L1). The co-propagating spontaneous Raman signal from the waveguide is coupled to the single-mode fibre (SMF) using another aspheric lens (L2) via a long pass edge filter (EF).

\subsection{Background luminescence from the waveguides}

The emission spectrum recorded with a setup depicted in figure 5 when a $785 \mathrm{~nm}$ laser is coupled to a $7 \mathrm{~mm}$ long waveguide $(\mathrm{w}=700 \mathrm{~nm}, \mathrm{~h}=220 \mathrm{~nm}$ ) is shown in figure 5 . The pump power was set to about $50 \mathrm{~mW}$ while the coupling loss is estimated to be approximately $10 \mathrm{~dB}$ per facet. A broad Raman emission in the range $2100-2400 \mathrm{~cm}^{-1}$ with a peak around $2330 \mathrm{~cm}^{-1}$ can be readily observed. Based on the observation of the peak with a commercial confocal microscope pumped at laser $532 \mathrm{~nm}$, as described in section 2, this peak can be concluded to be from the $\mathrm{Si}_{3} \mathrm{~N}_{4}$ material that forms the waveguide core. Another feature of the spectrum is the broad luminescence in the range below $1200 \mathrm{~cm}^{-1}$. From our preliminary studies on how it varies with the width of the waveguide, we suspect this is a predominantly (auto) fluorescence due to silica under-cladding. Our suspicion that this is not a Raman signal is also corroborated by the fact that it is not observed when we used confocal microscope with pump at wavelength $532 \mathrm{~nm}$. However, in addition to being broad, this part of the background spectrum remains practically constant for a given pump power, thus not posing a very serious difficulty for Raman spectroscopy. This must be mitigated, however, for Raman spectroscopy with a longer integration time.

\subsection{Observation of Raman spectra using single mode silicon-nitride waveguides}

We use a $\mathrm{Si}_{3} \mathrm{~N}_{4}$ waveguide $(\mathrm{w}=700 \mathrm{~nm}, \mathrm{~h}=220 \mathrm{~nm})$ on top of a $2.4 \mu \mathrm{m}$ silica cladding on a silicon substrate as our sensing platform (Figure 1) [5]. A spiral waveguide (1 cm in length) is used as the sensing region for the experiment (figure 6). The spiral forms the bottom of a rectangular reservoir defined lithographically using SU8. The laser power is set to $150 \mathrm{~mW}$, and coupled (coupling loss: 10 dB/facet) to the waveguide. For Raman measurements, two different concentrations of $7 \mu \mathrm{M}$ and $33 \mu \mathrm{M}$ of Rhodamine $6 \mathrm{G}$ (R4127, Sigma Aldrich) dye solution in DI water were prepared. A $3 \mu \mathrm{L}$ drop of the $7 \mu \mathrm{M}$ solution was first dispensed in the reservoir containing the waveguide spiral and covered with a thin glass coverslip to prevent water evaporation, and the Raman spectra is measured. Then, $33 \mu \mathrm{M}$ solution was then added on the same reservoir and measured (estimated concentration now being about $20 \mu \mathrm{M}$ ).

The measured spectra are shown in figure 7. A noticeable feature of the spectra is that they have the strongest peak at $2330 \mathrm{~cm}^{-1}$ corresponding to the $\mathrm{Si}_{3} \mathrm{~N}_{4}$ waveguide material, which was verified by z-scan using a commercial confocal 
Raman microscope, as described in section 2. A broad fluorescence background starting from about $1800 \mathrm{~cm}^{-1}$ and peaking up as we go lower in wavenumbers can also be seen in the spectra. This is due to auto-fluorescence of the silica under cladding, also described in section 4.1. The peaks, notably at $1360 \mathrm{~cm}^{-1}, 1515 \mathrm{~cm}^{-1}$ and $1600 \mathrm{~cm}^{-1}$ are due to the dye, which can be confirmed using the data provided by the manufacturer [7]. In addition to that we also observed the signal strength dependency on the concentration of the dye solution, with higher concentration (appox. $20 \mu \mathrm{M}$ ) yielding stronger signal than $7 \mu \mathrm{M}$ solution.

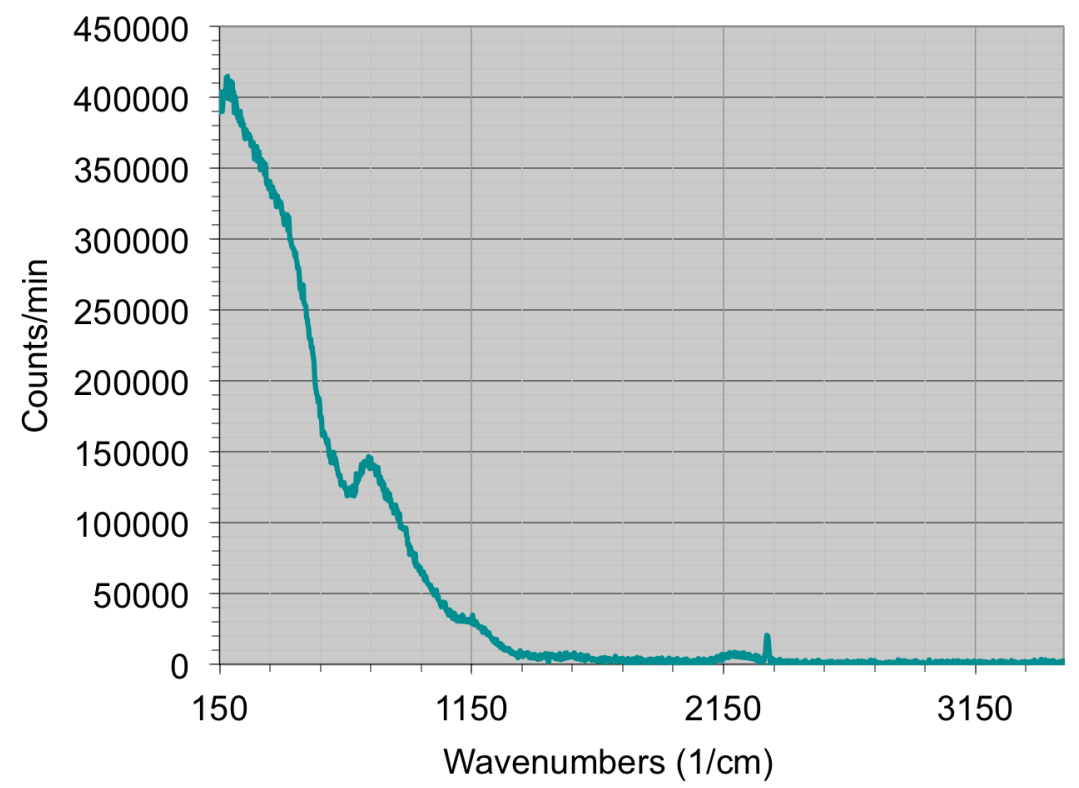

Figure 5: The luminescence spectrum recorded from a $7 \mathrm{~mm}$ waveguide using the setup described in figure 4 . The laser power was set to $50 \mathrm{~mW}$ and estimated coupling loss is about $10 \mathrm{~dB}$ per facet. We can clearly see a broad Raman emission in the range $2100-2400$ $\mathrm{cm}^{-1}$ with a peak around $2330 \mathrm{~cm}^{-1}$. This peak is also observed with commercial confocal microscope, as described in section 2 . The broad luminescence below $1200 \mathrm{~cm}^{-1}$ is a predominantly due to silica undercladding.

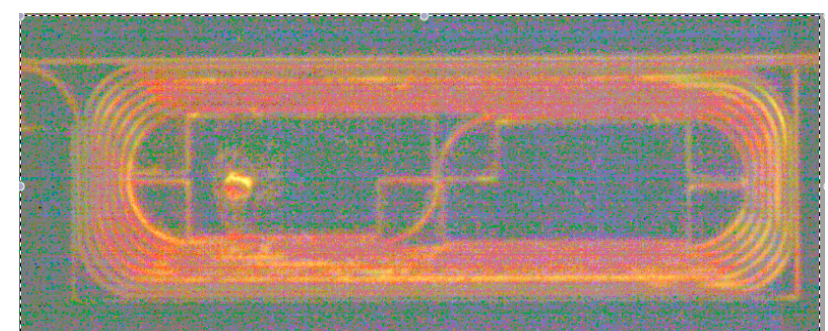

Figure 6: The spiral used for measurement tinted with R6G dye used as Raman active molecules.

\section{CONCLUSIONS}

We experimentally demonstrated for the first time, to the best of our knowledge, the measurement of Raman signal using single mode integrated optical waveguides whereby both the pump and the collection happen through the waveguide mode. We exploit the enhancement of light scattering due to the evanescent tail of the waveguide modes. We measured a Raman signal of approximately 300 counts/min for a $7 \mu \mathrm{M}$ concentration of Rodhamine $6 \mathrm{G}$ dye for a pump power of 150 $\mathrm{mW}$ and $10 \mathrm{~dB}$ coupling loss per facet. 


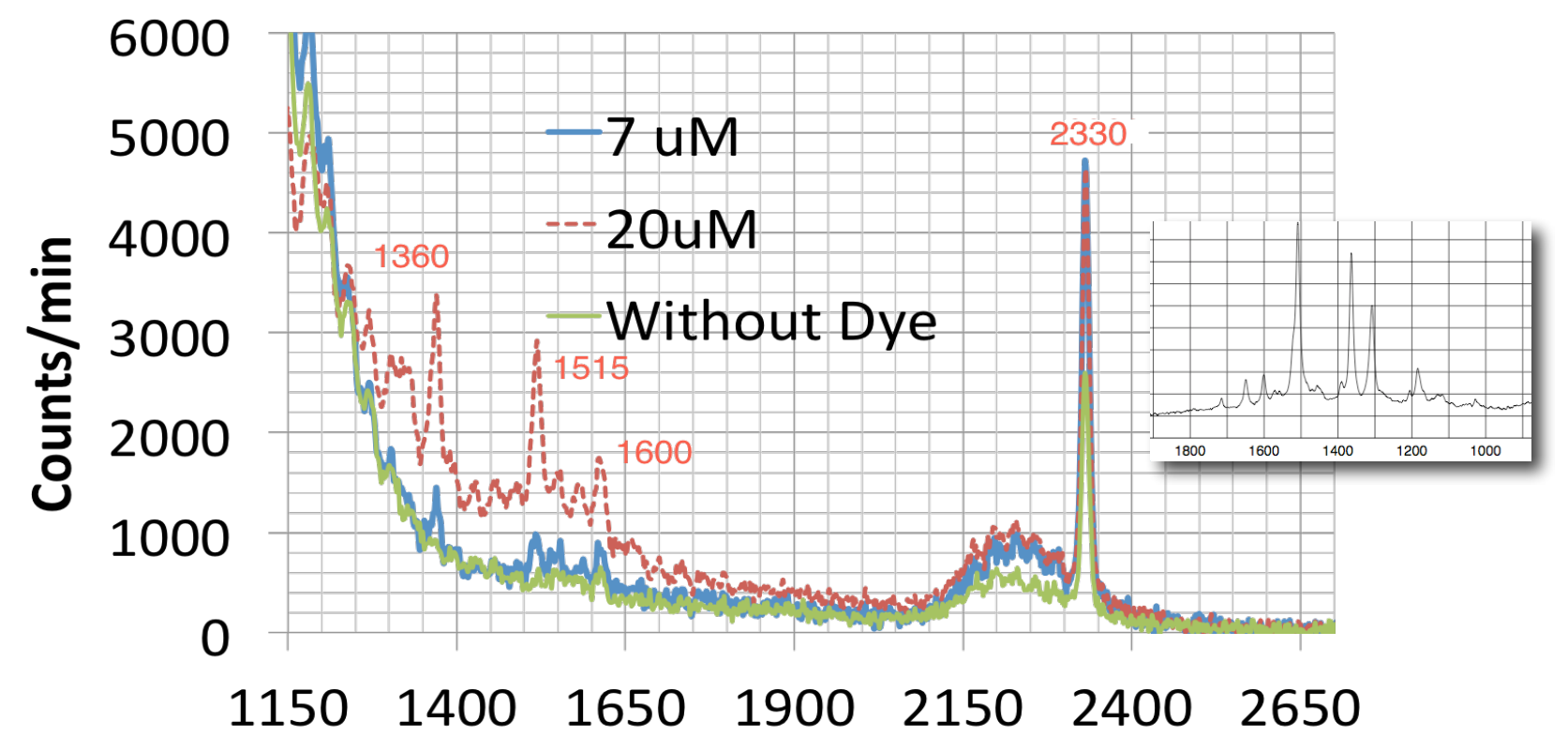

\section{Wavenumbers $(1 / \mathrm{cm})$}

Figure 7: Measured Raman spectrum for 7uM (Red) and 20uM (Blue) concentration of rhodamine 6G dye in water. The peaks at 1360 $\mathrm{cm}^{-1}, 1515 \mathrm{~cm}^{-1}$ and $1600 \mathrm{~cm}^{-1}$ correspond to that of dye while the at peak $2330 \mathrm{~cm}^{-1}$ corresponds to the $\mathrm{Si}_{3} \mathrm{~N}_{4}$ used as the waveguide core material. The green curve is without dye, with lower $(50 \mathrm{~mW})$ pump power. The Raman spectrum of the dye, as provided by the manufacturer [7] is also displayed on the inset for comparison.

\section{ACKNOWLEDGEMENT}

The authors acknowledge the ERC advanced grant InSpectra for the funding and imec, Leuven for processing of the waveguide.

\section{REFERENCES}

[1] J. Hubner, J., Jorgensen A.M., Anhoj, T.A., and Zauner, D. A., "Integrated optical systems for lab-on-chip applications," Proc. SPIE 5728, 269-277 (2005).

[2] Momeni, B., Yegnanarayanan, S., Soltani, M., Eftekhar, A. A., Shah Hosseini, E., and Adibi, A., "Silicon nanophotonic devices for integrated sensing," J. Nanophoton. 3(1), 031001 (2009).

[3] Dhakal. A.. Subramanivan. A. Z.. Thomas. N. L.. Baets R.. "The role of index contrast in the efficiencv of absorntion and emission of a luminescent particle near a slab waveguide", $16^{\text {th }}$ European Conference on Integrated Optics, Spain, 131 (2012).

[4] Dhakal, A., Subramaniyan, A. Z., Thomas, N. L., Baets R, "Enhancement of light emission, scattering, and emission in high index contrast waveguides" OSA Advanced Photonics Congress, Sensors , PuertoRico, ST2B.5, 2013

[5] Peyskens, F., Subramaniyan, A. Z., Dhakal, A., Thomas, N. L., Baets R., Enhancement of Raman Scattering Efficiency by a Metallic Nano-antenna on Top of a High Index Contrast Waveguide, CLEO 2013, United States, CM2F.5 (2013)

[6] Subramanian A Z. , et. al., "Low-loss singlemode PECVD silicon-nitride photonic wire waveguides for 532$900 \mathrm{~nm}$ wavelength window fabricated within a CMOS pilot line", IEEE Photonics Journal vol. 5, 2202809 (2013)

[7] www.sigmaaldrich.com/spectra/rair/RAIR003657.PDF 